\title{
Vitamin C intake in relation to bone mineral density and risk of hip fracture and osteoporosis: a systematic review and meta-analysis of observational studies
}

\author{
Hanieh Malmir ${ }^{1,2}$, Sakineh Shab-Bidar ${ }^{2 *}$ and Kurosh Djafarian ${ }^{3}$ \\ ${ }^{1}$ Students' Scientific Research Center, Tebran University of Medical Sciences, PO Box 14177-55331, Tehran, Iran \\ ${ }^{2}$ Department of Community Nutrition, School of Nutritional Sciences and Dietetics, Tehran University of Medical Sciences, \\ PO Box 14155-6117, Tehran, Iran \\ ${ }^{3}$ Department of Clinical Nutrition, School of Nutritional Sciences and Dietetics, Tehran University of Medical Sciences, PO Box \\ 14155-6117, Tehran, Iran
}

(Submitted 1 July 2017 - Final revision received 22 December 2017 - Accepted 12 January 2018)

\section{Abstract}

We aimed to systematically review available data on the association between vitamin C intake and bone mineral density (BMD), as well as risk of fractures and osteoporosis, and to summarise this information through a meta-analysis. Previous studies on vitamin $\mathrm{C}$ intake in relation to BMD and risk of fracture and osteoporosis were selected through searching PubMed, Scopus, ISI Web of Science and Google Scholar databases before February 2017, using MeSH and text words. To pool data, either a fixed-effects model or a random-effects model was used, and for assessing heterogeneity, Cochran's $Q$ and $I^{2}$ tests were used. Subgroup analysis was applied to define possible sources of heterogeneity. Greater dietary vitamin C intake was positively associated with BMD at femoral neck (pooled $r 0 \cdot 18 ; 0 \cdot 06,0 \cdot 30$ ) and lumbar spine (pooled $r$ 0.14; $95 \%$ CI 0.06, 0.22); however, significant between-study heterogeneity was found at femoral neck: $I^{2}=87 \cdot 6 \%$, $P_{\text {heterogeneity }}<0.001$. In addition, we found a non-significant association between dietary vitamin C intake and the risk of hip fracture (overall relative risk $=0.74 ; 95 \%$ CI $0.51,1 \cdot 08)$. Significant between-study heterogeneity was found $\left(I^{2}=79 \cdot 1 \%, P_{\text {heterogeneity }}<0 \cdot 001\right)$, and subgroup analysis indicated that study design, sex and age were the main sources of heterogeneity. Greater dietary vitamin C intake was associated with a $33 \%$ lower risk of osteoporosis (overall relative risk $=0.67 ; 95 \%$ CI 0.47, 0.94). Greater dietary vitamin C intake was associated with a lower risk of hip fracture and osteoporosis, as well as higher BMD, at femoral neck and lumbar spine.

\section{Keywords: Vitamin C: Fractures: Bone mineral density: Osteoporosis: Meta-analyses}

Osteoporosis is a chronic condition that results in reducing bone density and increasing bone fragility ${ }^{(1)}$. Osteoporosis is generally diagnosed with bone mineral density (BMD) measurement by dual-energy X-ray absorptiometry (DXA) ${ }^{(2)}$. The World Health Organization ${ }^{(3)}$ defines osteoporosis as BMD T-score equal to or lower than $-2 \cdot 5^{(3)}$. In most patients, osteoporosis has no symptoms until a fracture occurs. Osteoporosis causes nearly nine million fractures annually worldwide and more than half of them occur in America and Europe ${ }^{(4)}$. On the basis of the severity and location of fractures, many complications, including significant disability, increased dependency, reduced quality of life and increased economic burden of healthcare costs, may occur ${ }^{(4-7)}$.

Age, sex, ethnicity, family history, fracture history, some diseases and disorders (such as hypo-gonadal states, endocrine disorders, malnutrition, rheumatologic disorders, renal insufficiency and haematologic disorders), alcohol consumption, tobacco smoking and lack of physical activity are some of the risk factors for osteoporosis and fractures ${ }^{(8-15)}$. Dietary and nutritional factors have been identified as having a role in the prevention and incidence of osteoporosis and fractures. On the basis of previous studies, low intake of $\mathrm{Ca}, \mathrm{P}, \mathrm{Mg}, \mathrm{Zn}, \mathrm{B}, \mathrm{Fe}$, fluoride, $\mathrm{Cu}$, vitamins $\mathrm{A}, \mathrm{K}$ and $\mathrm{D}$ and high intake of $\mathrm{Na}$, animal protein and soft drinks may result in osteoporosis and fractures $^{(16-18)}$. Vitamin $C$ is one of the dietary components that affect BMD. Vitamin C affects collagen synthesis and osteoblast genesis. Earlier studies have shown an inverse relationship between vitamin $\mathrm{C}$ intake and the risk of fracture or osteoporosis $^{(19-21)}$. However, one cohort study of 4367 people aged 39-79 years reached no significant association ${ }^{(22)}$. In addition, increased vitamin $\mathrm{C}$ intake was associated with higher BMD at different sites ${ }^{(23-29)}$. Other studies have failed to find any significant association between vitamin $\mathrm{C}$ intake and $\mathrm{BMD}^{(30,31)}$.

Given the conflicting findings, this study aimed to systematically review available data on the association between vitamin C intake and BMD, as well as risk of fractures and osteoporosis, and to summarise this information by performing a meta-analysis.

Abbreviations: BMD, bone mineral density; RR, relative risk.

* Corresponding author: S. Shab-Bidar, fax +98 21 88984861, email s-shabbidar@tums.ac.ir 


\section{Methods}

This systematic review and meta-analysis was performed based on Preferred Reporting Items for Systematic Reviews and Meta-Analyses (PRISMA) guidelines ${ }^{(32)}$ and has been recorded in PROSPERO by 42017055780 ID number.

\section{Search strategy}

Previous observational studies of vitamin $\mathrm{C}$ intake in relation to BMD or risk of fracture or osteoporosis were selected through searching PubMed, ISI Web of Science and Google Scholar before February 2017 by two reviewers independently (H. M., S. S.-b.). We used the following keywords in the search: ('vitamin C'[tiab] OR 'ascorbic acid'[tiab] OR 'acid ascorbic'[tiab] OR ascorbate[tiab] OR 'ascorbic acid'[MeSH]) AND ('bone mineral density'[tiab] OR 'bone mass density'[tiab] OR 'bone density'[tiab] OR BMD[tiab] OR fracture[tiab] OR osteoporosis [tiab] OR 'bone density'[MeSH] OR 'fractures, bone'[MeSH] OR osteoporosis[MeSH]). No limitation was applied during the search. The reference lists of retrieved papers were also examined to avoid missing any published data.

\section{Inclusion criteria}

Publications that fulfilled the following criteria were eligible for inclusion: (1) all studies, conducted on humans, that examined the relationship between vitamin $\mathrm{C}$ intake and BMD, risk of fractures and osteoporosis; (2) studies that were of crosssectional or case-control or cohort design; (3) those that reported OR or hazards ratios (HR) along with $95 \%$ CI for fracture and osteoporosis; and (4) those that reported correlation coefficient for BMD.

Studies were excluded because of the following reasons: (1) those that were letters, comments, reviews, meta-analyses, ecological studies, animal studies and clinical trial studies; (2) studies that did not report any estimates for the association between vitamin $\mathrm{C}$ intake and the intended outcomes; and (3) studies that examined the relationship in children and adolescents. When we found more than one published report based on the same study population ${ }^{(33)}$, only the most comprehensive publication was included in this meta-analysis ${ }^{(34)}$.

\section{Data extraction}

From each eligible study, the following information was extracted: first author, year of publication, study design, country, age range, sex, sample size, number of cases, duration of follow-up, exposure variable, assessment of exposure, outcome variable, assessment of outcome, relevant effect sizes (OR or HR and $95 \% \mathrm{CI}$, correlation coefficient), methods of fracture or BMD quantification and covariates adjusted.

\section{Quality assessment}

The quality of included studies was examined using the Newcastle-Ottawa Scale (NOS) by two reviewers independently (H. M. and S. S.-b.) ${ }^{(35)}$. The NOS assigns a maximum of nine points to each study: four for selection, two for comparability and three for assessment of outcomes and exposures.
In the current analysis, when a study got more than median stars, it was considered as relatively high quality; otherwise, it was deemed to have low quality. All included studies were agreed upon.

\section{Statistical methods}

The effect sizes that we used in this analysis were HR and OR and their $95 \%$ CI for risk of fracture and osteoporosis in the highest $v$. the lowest category of vitamin $\mathrm{C}$ intake. In addition, the correlation coefficient between vitamin $\mathrm{C}$ intake and BMD was used. For correlation analysis, Fisher's $Z$ and sample size was calculated and used to conduct the meta-analysis. A fixedeffect model was used to calculate pooled risk estimates by generic inverse variance method by the user-written 'metan' command in Stata (version 14) software ${ }^{(36)}$. Heterogeneity was assessed using Cochrane's $Q$ test, and $I^{2}$ statistic provided the relative amount of variance of the summary effect ${ }^{(37)}$. In cases with heterogeneity, random-effects model (DerSimonian-Laird) was used. To evaluate the predefined sources of heterogeneity, subgroup analyses were conducted using a fixed-effect model with the user-written 'metan' command ('by option') in Stata (version 14) software ${ }^{(36)}$. Publication bias was assessed by visual inspection of funnel plots. Tests for funnel plot asymmetry were done with the user-written 'metabias' command in Stata (version 11) software 31. Statistical analyses were carried out by the use of STATA, version 14.0 (StataCorp). $P$ values that were $<0.1$ for heterogeneity test and $<0.05$ in others were considered statistically significant.

\section{Results}

\section{Findings from a systematic review}

In total, 1462 articles were found in our initial search. After screening titles and abstracts, 1423 articles were excluded. After these exclusions, thirty-eight articles remained for systematic review and twelve studies were included in the meta-analysis. The details of the study selection process are shown in Fig. 1.

We included thirty-eight studies (ten cohort ${ }^{(20,22,34,38-44)}$, seven case-control ${ }^{(19,26,45-49)}$ and twenty-one crosssectional $\left.^{(21,23-25,27-31,50-61)}\right)$ in the systematic review (Tables 1-3). These studies involved 106741 individuals aged 20-103 years. All studies were published between 1988 and 2016. In all, fourteen studies were conducted in $\mathrm{USA}^{(20,28,29,31,39,42,44,48-50,52,55,58,59)}$, thirteen in East Asian countries and New Zealand ${ }^{(19,21,23-26,30,38,43,56,57,60,61)}$ and eleven in European countries ${ }^{(22,27,34,40,41,45-47,51,53,54)}$. Two studies were conducted on males ${ }^{(38,60)}$, fourteen on both males and females ${ }^{(19-21,24,27,39-42,44,46,49,52,55)}$ and others on females ${ }^{(23,25,26,29-31,34,45,47,48,50,53,54,57-59,61)}$. Three studies had considered osteoporosis as the outcome ${ }^{(21,43,61)}$; twelve studies examined fracture as the outcome ${ }^{(19,20,22,39-41,44-49)}$; and twenty-two studies assessed BMD as the outcome ${ }^{(23-25,27-31,34,38,50-60)}$. One study had reported both BMD and fracture as the main outcomes ${ }^{(42)}$ and two studies had reported both osteoporosis and BMD as the main outcomes $^{(26,61)}$. The duration of follow-up in cohort 


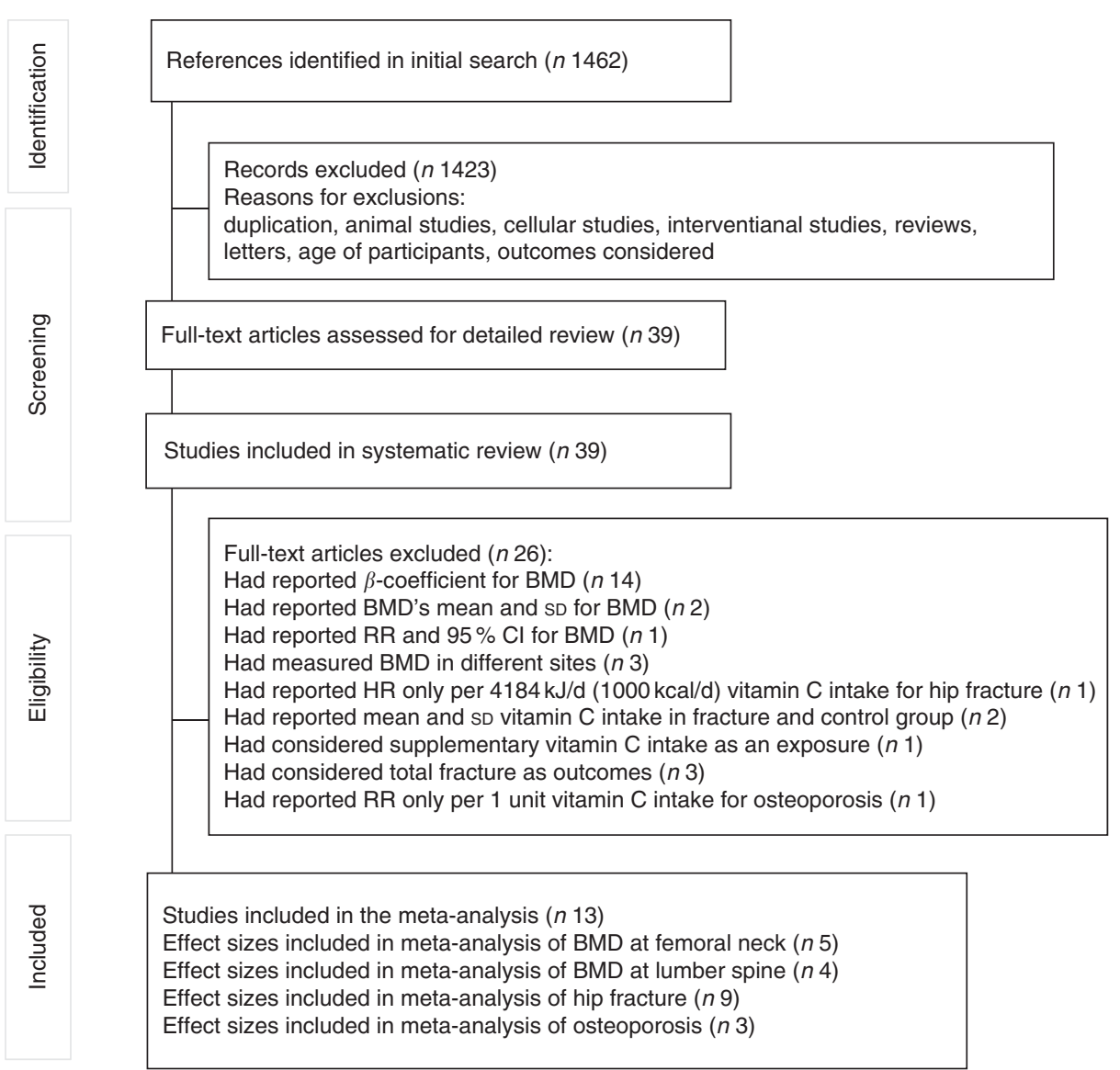

Fig. 1. Flow chart of article searching. BMD, bone mineral density; RR, relative risk; HR, hazard ratio.

studies was between $4^{(38,43)}$ and 20 years ${ }^{(44)}$. Four studies used supplementary vitamin $\mathrm{C}$ intake as exposure ${ }^{(20,44,52,55)}$, four studies used total dietary and supplementary vitamin $C$ intakes as exposure $e^{(20,31,50,59)}$ and in other studies dietary vitamin $\mathrm{C}$ intakes have been used as exposure $^{(19-31,34,38-43,45-51,53-61)}$. In seven studies, 24-h dietary recall for dietary assessment ${ }^{(21,25,39,42,45,54,60)}$, in four studies food records ${ }^{(22,27,28,51)}$, questionnaire for three studies ${ }^{(44,52,58)}$, in one diet history ${ }^{(56)}$ and in other studies validated FFQ $^{(19,20,23,24,26,29-31,34,38,40,41,43,46-50,53,55,57,59,61)}$ were used to record exposure.

BMD was measured at different sites in included studies; fourteen studies have quantified BMD in femoral neck $^{(23-27,29,31,34,38,52,55,58-60)}$, seven at lumbar spine ${ }^{(23,25,26,29,34,52,58)}$, nine at total hip ${ }^{(23-25,27,29,34,38,51,52)}$ and three at trochanter ${ }^{(27,28,55)}$. BMD was also measured in ultradistal radius ${ }^{(50,52)}$, calcaneus ${ }^{(54,56,61)}$, total forearm, trabecular forearm, cortical forearm ${ }^{(53)}$, midshaft radius ${ }^{(51)}$, proximal femur ${ }^{(42)}$, total femur, shaft, $\operatorname{ward}^{(28)}$, whole body ${ }^{(27,30)}$, fore$\operatorname{arm}^{(57)}$ and femoral total ${ }^{(26)}$. All studies used DXA for measuring BMD, except two studies that used single-photon absorptiometry ${ }^{(50,52)}$ and one that used qualitative ultrasound (QUS) ${ }^{(61)}$. To assess BMD, eleven studies applied Hologic scanner ${ }^{(24,25,27,29-31,38,51,59-61)}$, five applied Lunnar scanner ${ }^{(23,26,28,54,55)}$, two studies applied XR-26 scanner ${ }^{(40,53)}$, one study applied ARKRAY scanner ${ }^{(56)}$, one study applied ALOKA scanner ${ }^{(57)}$ and one did not report the measurement tool ${ }^{(42)}$. In terms of site of fracture, nine studies considered hip $^{(19,20,22,39,44,45,47-49)}$, four considered total fracture ${ }^{(40,411,42,46)}$, two studies considered spine $e^{(22,44)}$ and one study considered wrist fracture ${ }^{(44)}$. In terms of osteoporosis, three studies measured BMD T-score by DXA ${ }^{(21,26,43)}$ and one study measured BMD T-score by QUS ${ }^{(61)}$.

In terms of BMD, increased vitamin $\mathrm{C}$ intake was associated with higher BMD at different sites in sixteen studies $(23-29,34,42,46,52,54,55,57,60,61)$. However, no significant association was seen between vitamin C intake and BMD at different sites in other studies $30,31,38,51,53,56,58,59)$. Higher vitamin $C$ intake was associated with a reduced risk of fracture in two studies $^{(19,54)}$. No significant association was found in other studies $^{(22,39,40,42,44,46-49)}$. Osteoporosis was directly associated with dietary vitamin $C$ intake in one study ${ }^{(61)}$ and inversely associated with dietary vitamin $\mathrm{C}$ intake in another study ${ }^{(21)}$. However, two studies did not find any significant association between dietary vitamin $\mathrm{C}$ intake and the risk of osteoporosis ${ }^{(26,43)}$.

\section{Findings of the meta-analysis}

Vitamin C intake and bone mineral density. Out of twentyfour studies that were included in the systematic review, we excluded twenty studies because of the following reasons: those that reported results as beta regression 


\section{NS British Journal of Nutrition}

Table 1. Characteristics of studies that reported the relationship between vitamin $C$ intake in relation to bone mineral density (BMD)

( $\beta$-Coefficients with their standard errors; mean values and standard deviations; odds ratios and $95 \%$ confidence intervals)

\begin{tabular}{|c|c|c|c|c|c|c|c|c|c|c|c|c|c|c|c|c|c|c|c|}
\hline \multirow{3}{*}{$\begin{array}{l}\text { Author (year) } \\
\text { (references) }^{\text {Hernandez-Avile }} \\
\text { (1992) }\end{array}$} & \multirow{3}{*}{$\begin{array}{l}\text { Study design } \\
\text { Cross-sectional }\end{array}$} & \multirow{3}{*}{$\frac{\text { Country }}{\text { USA }}$} & \multirow{3}{*}{$\begin{array}{c}\begin{array}{c}\text { Age range } \\
\text { (years) }\end{array} \\
50-60\end{array}$} & \multirow{3}{*}{$\frac{\operatorname{Sex}}{\mathrm{F}}$} & \multirow{3}{*}{$\frac{n}{281}$} & \multirow{3}{*}{$\begin{array}{c}\begin{array}{c}\text { Duration of } \\
\text { follow-up } \\
\text { (years) }\end{array} \\
-\end{array}$} & \multirow{3}{*}{$\begin{array}{l}\text { Exposure } \\
\text { variable } \\
\text { Dietary vitamin C } \\
\text { Total vitamin C }\end{array}$} & \multirow{3}{*}{$\begin{array}{l}\begin{array}{l}\text { Exposure } \\
\text { assessment }\end{array} \\
\text { FFQ }\end{array}$} & \multirow{2}{*}{\multicolumn{2}{|c|}{ Outcome variable }} & \multirow[b]{2}{*}{ Outcome assessment } & \multirow[b]{2}{*}{ Comparison } & \multicolumn{5}{|c|}{ Result } & \multirow[b]{2}{*}{$\begin{array}{l}\text { Study } \\
\text { quality }\end{array}$} & \multirow[b]{2}{*}{ Matching or adjustments } \\
\hline & & & & & & & & & & & & & $R$ & $\beta$ & SE & $\begin{array}{l}\text { Mean or } \\
\text { OR }\end{array}$ & $\begin{array}{l}\text { so or } \\
95 \% \mathrm{Cl}\end{array}$ & & \\
\hline & & & & & & & & & BMD & Ultra-distal radius & $\begin{array}{l}\text { Single-photon } \\
\text { absorptiometry } \\
\text { (Lunar) }\end{array}$ & Per 1 unit & & $\begin{array}{l}0.086 \\
0.025\end{array}$ & $\begin{array}{l}0.056 \\
0.013\end{array}$ & & & 8 & $\begin{array}{l}\text { Age, Ca intake, smoking, BMI, multi- } \\
\text { vitamin intake, luteinising hormone, } \\
\text { follicle-stimulating hormone }\end{array}$ \\
\hline Leveille $(1997)^{(31)}$ & Cross-sectional & USA & $55-80$ & $\mathrm{~F}$ & 1892 & - & $\begin{array}{l}\text { Dietary vitamin } C \\
\text { Total vitamin C }\end{array}$ & $\mathrm{FFQ}$ & BMD & Femoral neck & DXA (Hologic) & Per 1 unit & & $\begin{array}{l}0.010 \\
0.005\end{array}$ & $\begin{array}{l}0.044 \\
0.006\end{array}$ & & & 9 & $\begin{array}{l}\text { Age, weight, height, thiazide use, } \\
\text { thyroid use, energy intake, physical } \\
\text { activity, vitamin } \mathrm{D} \text { and } \mathrm{E}, \mathrm{Ca} \text {, } \\
\beta \text {-carotene intake, diabetes, } \\
\text { oestrogen use }\end{array}$ \\
\hline New $(1997)^{(33)}$ & Cross-sectional & UK & $45-49$ & $\mathrm{~F}$ & 994 & - & Dietary vitamin C & $\mathrm{FFQ}$ & BMD & $\begin{array}{l}\text { Lumbar spine } \\
\text { Femoral neck } \\
\text { Femoral trochanter } \\
\text { Femoral wards }\end{array}$ & DXA (XR-26) & - & $\begin{array}{l}0.07 \\
0.03 \\
0.05 \\
0.05\end{array}$ & & & & & 7 & $\begin{array}{l}\text { Age, weight, height, physical activity, } \\
\text { smoking, social status }\end{array}$ \\
\hline Wang $(1997)^{(58)}$ & Cross-sectional & USA & $59-84$ & $\mathrm{~F}$ & 125 & - & Dietary vitamin C & Questionnaire & BMD & $\begin{array}{l}\text { Femoral neck } \\
\text { Lumbar spine }\end{array}$ & DXA (Hologic) & Per 1 unit & & $\begin{array}{l}0.0002 \\
0.0002\end{array}$ & $\begin{array}{l}0.0001 \\
0.002\end{array}$ & & & 4 & $\begin{array}{l}\text { Age, BMI, energy intake, oestrogen } \\
\text { use, acculturation, moderate activity }\end{array}$ \\
\hline Hall (1998) & Cross-sectional & USA & $45-65$ & $\mathrm{~F}$ & 775 & - & Dietary vitamin C & $\mathrm{FFQ}$ & BMD & $\begin{array}{l}\text { Femoral neck } \\
\text { Total hip } \\
\text { Lumbar spine }\end{array}$ & DXA (Hologic) & Per $100 \mathrm{mg} / \mathrm{d}$ & & & & & & 7 & $\begin{array}{l}\text { Age, BMI, oestrogen use, energy } \\
\text { intake, Ca intake, physical activity, } \\
\text { smoking }\end{array}$ \\
\hline New $(2000)^{(53)}$ & Cross-sectional & UK & $45-55$ & $\mathrm{~F}$ & 62 & - & Dietary vitamin C & $\mathrm{FFQ}$ & BMD & $\begin{array}{l}\text { Total forearm } \\
\text { Trabecular forearm } \\
\text { Cortical forearm }\end{array}$ & DXA (XR-26) & - & $\begin{array}{l}0.11 \\
0.09 \\
0.16\end{array}$ & & & & & 7 & $\begin{array}{l}\text { Age, weight, height, menopausal } \\
\text { status }\end{array}$ \\
\hline Morton $(2001)^{(52)}$ & Cross-sectional & USA & $50-98$ & $\mathrm{~F}$ & 994 & - & $\begin{array}{l}\text { Supplementary } \\
\text { vitamin C }\end{array}$ & Questionnaire & BMD & $\begin{array}{l}\text { Ultra-distal radius } \\
\text { Midshaft radius } \\
\text { Femoral neck } \\
\text { Total hip } \\
\text { Lumbar spine }\end{array}$ & $\begin{array}{l}\text { Single-photon } \\
\text { absorptiometry } \\
\text { (Lunar) }\end{array}$ & $\begin{array}{l}\text { Users } v \text {. } \\
\text { non-users }\end{array}$ & & & & \begin{tabular}{l|l}
0.247 & \\
0.237 & \\
0.587 & \\
0.586 & \\
0.656 & \\
0.630 & \\
0.803 & \\
0.788 & \\
0.925 & \\
0.907 &
\end{tabular} & $\begin{array}{l}0.235,0.259 \\
0.231,0.243 \\
0.571,0.603 \\
0.576,0.596 \\
0.639,0.674 \\
0.620,0.639 \\
0.782,0.824 \\
0.767,0.789 \\
0.896,0.954 \\
0.890,0.924\end{array}$ & 7 & $\begin{array}{l}\text { Age, BMI, exercise, alcohol, smoking, } \\
\text { Ca and multi-vitamin supplement, } \\
\text { thiazide, oestrogen, thyroid hormone, } \\
\text { corticosteroid use, physical activity }\end{array}$ \\
\hline Sasaki $(2001)^{(56)}$ & Cross-sectional & Japan & $\begin{array}{l}29-60 \\
39-60\end{array}$ & $\mathrm{~F}$ & $\begin{array}{l}\text { Pre: } 243 \\
\text { Pos: } 137\end{array}$ & - & Dietary vitamin C & Diet history & BMD & Calcaneus & DXA (ARKRAY) & & $\begin{array}{l}0.07 \\
0.10\end{array}$ & & & $\begin{array}{l}129 \\
154\end{array}$ & $\begin{array}{l}83 \\
73\end{array}$ & 4 & $\begin{array}{l}\text { Age, height, fat and non-fat body } \\
\text { weight, energy intake }\end{array}$ \\
\hline Simon $(2001)^{(42)}$ & Cohort & USA & $20-90$ & $\begin{array}{l}F \\
M\end{array}$ & $\begin{array}{r}\text { Pre: } 3204 \\
\text { Pos: } 2906 \\
5739\end{array}$ & 6 & Dietary vitamin $C$ & 24-h recall & BMD & Proximal femur & $\begin{array}{l}\text { DXA } \\
-\end{array}$ & Per $100 \mathrm{mg} / \mathrm{d}$ & & $\begin{array}{r}0 \\
0 \\
-0\end{array}$ & & & & 7 & $\begin{array}{l}\text { Age, race, education, physical activity, } \\
\text { BMI, thiazide, energy, fat, protein, } \\
\text { caffeine, alcohol intake, smoking, } \\
\text { diabetes, serum levels of vitamins E } \\
\text { and D and thyroid-stimulating } \\
\text { hormone, oral contraceptive pill, } \\
\text { pregnancy, oestrogen }\end{array}$ \\
\hline Ilich (2003) & Cross-sectional & USA & $57-88$ & $\mathrm{~F}$ & 136 & - & Dietary vitamin C & 3-d food record & BMD & $\begin{array}{l}\text { Total femur } \\
\text { Shaft } \\
\text { Trochanter } \\
\text { Ward }\end{array}$ & DXA (Lunar) & Per 1 unit & & $\begin{array}{l}0.4 \\
0 . \\
0 . c \\
0.6\end{array}$ & & & & 5 & $\begin{array}{l}\text { Age, lean body mass, total body fat, } \\
\text { height, physical activity, energy } \\
\text { intake }\end{array}$ \\
\hline Kaptoge $(2003)^{(51)}$ & Cross-sectional & UK & $67-79$ & $\begin{array}{l}\mathrm{F} \\
\mathrm{M}\end{array}$ & $\begin{array}{l}474 \\
470\end{array}$ & - & Dietary vitamin C & 7-d food record & BMD & Total hip & DXA (Hologic) & Per 1 unit & & $\begin{array}{l}0.159 \\
0.065\end{array}$ & $\begin{array}{l}0.112 \\
0.123\end{array}$ & & & 6 & $\begin{array}{l}\text { Weight change, physical activity, FEV, } \\
\text { ADL score }\end{array}$ \\
\hline Macdonald (2004) & Cohort & UK & $45-55$ & $\mathrm{~F}$ & 891 & 5 & Dietary vitamin C & $\mathrm{FFQ}$ & BMD & $\begin{array}{l}\text { Femoral neck } \\
\text { Lumbar spine }\end{array}$ & DXA (XR-26) & & $\begin{array}{l}0.195 \\
0.104\end{array}$ & & & & & 7 & $\begin{array}{l}\text { Age, weight, weight change, height, } \\
\text { smoking, physical activity, socio- } \\
\text { economic status }\end{array}$ \\
\hline Wolf $(2005)^{(59)}$ & Cross-sectional & USA & $50-79$ & $\mathrm{~F}$ & 11068 & - & $\begin{array}{l}\text { Dietary vitamin C } \\
\text { Total vitamin C }\end{array}$ & $\mathrm{FFQ}$ & BMD & Femoral neck & DXA (Hologic) & Per 1 unit & & $\begin{array}{l}0.008 \\
0.001\end{array}$ & $\begin{array}{l}0.004 \\
0.001\end{array}$ & & & 7 & $\begin{array}{l}\text { Age, BMI, waist circumference, race, } \\
\text { income, education, alcohol, coffee, } \\
\text { smoking, hormone use, thiazide use, } \\
\text { thyroid medication, physical activity, }\end{array}$ \\
\hline
\end{tabular}




\section{N British Journal of Nutrition}

Table 1. Continued

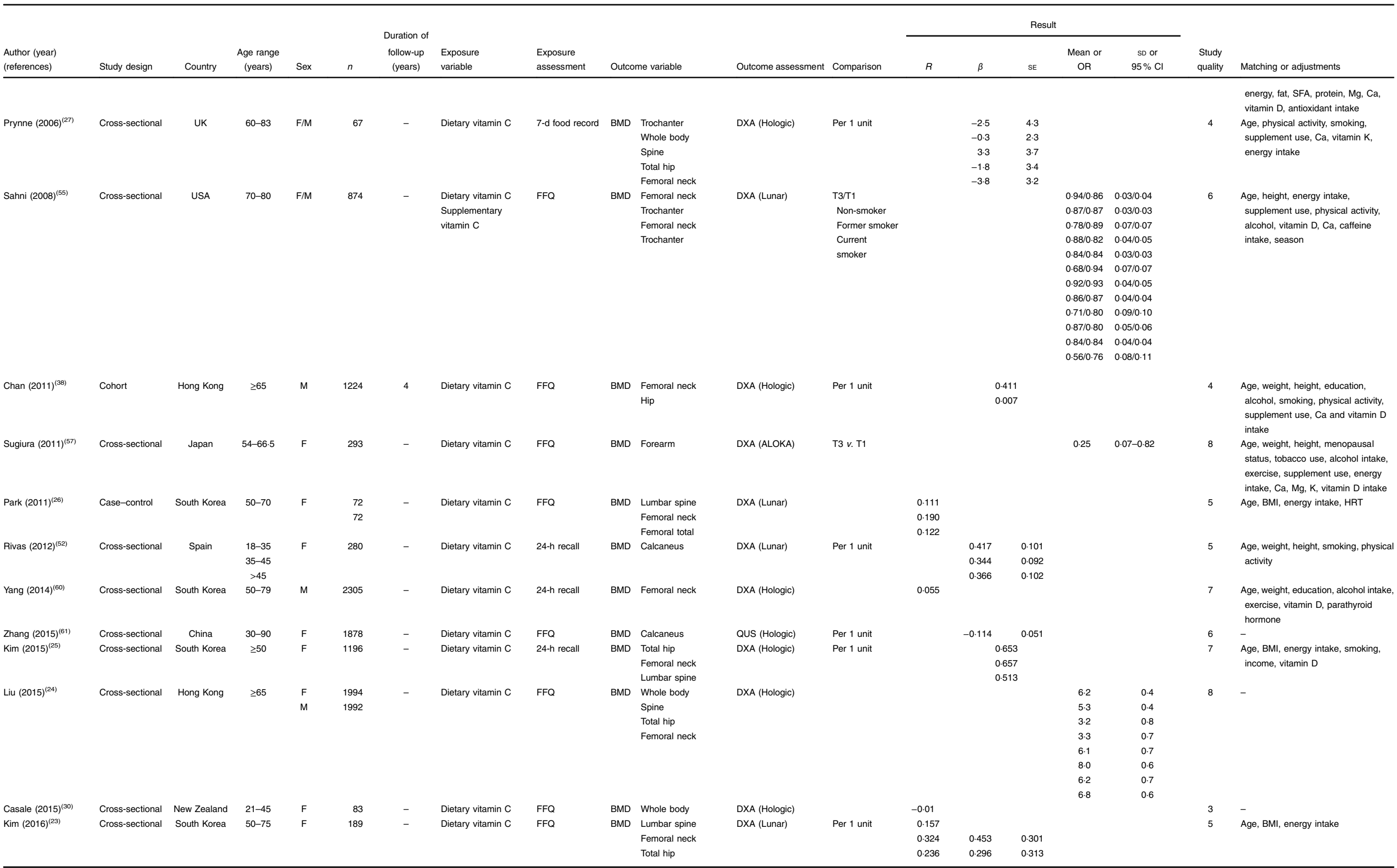

DXA, dual-energy X-ray absorptiometry; F, female; M, male; HRT, hormone replacement therapy; QUS, qualitative ultrasound. 
Table 2. Characteristics of studies that reported the relationship between vitamin $C$ intake and risk of fracture (Odds ratios, relative risks (RR) and $95 \%$ confidence intervals; mean values and standard deviations)

\begin{tabular}{|c|c|c|c|c|c|c|c|c|c|c|c|c|c|c|c|c|c|c|}
\hline First author (year) & $\begin{array}{l}\text { Study } \\
\text { design }\end{array}$ & Country & $\begin{array}{l}\text { Age range } \\
\text { (years) }\end{array}$ & Sex & $n$ & $\begin{array}{l}\text { Number of } \\
\text { cases }\end{array}$ & $\begin{array}{l}\text { Duration of follow- } \\
\text { up (years) }\end{array}$ & Exposure variable & $\begin{array}{l}\text { Exposure } \\
\text { assessment }\end{array}$ & $\begin{array}{l}\text { Outcome } \\
\text { variable }\end{array}$ & $\begin{array}{l}\text { Outcome } \\
\text { assessment }\end{array}$ & Comparison & $\begin{array}{c}\text { OR or } \\
\mathrm{RR}\end{array}$ & $95 \% \mathrm{Cl}$ & Mean & SD & $\begin{array}{l}\text { Study } \\
\text { quality }\end{array}$ & Matching or adjustments \\
\hline Holbrook $(1988)^{(39)}$ & Cohort & USA & 50-79 & $F / M$ & 957 & 33 & 14 & $\begin{array}{l}\text { Dietary vitamin C } \\
\text { intake }\end{array}$ & 24-h recall & Hip fracture & Interview & $\begin{array}{l}\text { Per } 48.6 \mathrm{mg} / 4184 \mathrm{~kJ} \\
(1000 \mathrm{kcal})\end{array}$ & 0.8 & NS & & & 5 & Age, sex, Ca \\
\hline Nieves $(1992)^{(48)}$ & $\begin{array}{l}\text { Case- } \\
\text { control }\end{array}$ & USA & $50-103$ & $\mathrm{~F}$ & 329 & 161 & - & $\begin{array}{l}\text { Dietary vitamin C } \\
\text { intake }\end{array}$ & FFQ & Hip fracture & $\begin{array}{l}\text { Radiology } \\
\text { photography }\end{array}$ & Q5 v. Q1 & 0.87 & $0 \cdot 34,2 \cdot 20$ & & & 6 & $\begin{array}{l}\text { BMI, oestrogen use, chronic } \\
\text { disease/age and hospital matching }\end{array}$ \\
\hline Michaelsson $(1995)^{(47)}$ & $\begin{array}{l}\text { Case- } \\
\text { control }\end{array}$ & Sweden & $40-75$ & $\mathrm{~F}$ & 1140 & 247 & - & $\begin{array}{l}\text { Dietary vitamin C } \\
\text { intake }\end{array}$ & $\mathrm{FFQ}$ & Hip fracture & Hospital record & Q4 v. Q1 & 1.89 & $1.15,3.09$ & & & 7 & $\begin{array}{l}\text { Diabetes, fracture history, HRT, } \\
\text { smoking, physical activity, BMI, } \\
\text { energy intake }\end{array}$ \\
\hline Lumbers $(2001)^{(45)}$ & $\begin{array}{l}\text { Case- } \\
\text { control }\end{array}$ & UK & $\geq 60$ & $\mathrm{~F}$ & 125 & 75 & - & $\begin{array}{l}\text { Dietary vitamin C } \\
\text { intake }\end{array}$ & 24-h recall & Hip fracture & Medical record & $\begin{array}{l}\text { fracture } \\
\text { control }\end{array}$ & & & $\begin{array}{l}60.7 \\
55.2\end{array}$ & $\begin{array}{l}33.2 \\
38.8\end{array}$ & & - \\
\hline Simon $(2001)^{(42)}$ & Cohort & USA & $20-90$ & $\mathrm{~F}$ & $\begin{array}{l}\text { Pre: } 3778 \\
\text { Pos: } 3165\end{array}$ & $\begin{array}{l}186 \\
392\end{array}$ & 6 & $\begin{array}{l}\text { Dietary vitamin C } \\
\text { intake }\end{array}$ & 24-h recall & Fracture & Interview & Per $100 \mathrm{mg} / \mathrm{d}$ & $\begin{array}{l}0.89 \\
0.99\end{array}$ & $\begin{array}{r}0.6,1 \cdot 32 \\
0.83,1 \cdot 17\end{array}$ & & & 6 & $\begin{array}{l}\text { Age, race, education, physical } \\
\text { activity, BMI, oral contraceptive pill, } \\
\text { thiazide diuretics, history of } \\
\text { pregnancy, Ca, fat, protein, caffeine, } \\
\text { alcohol intake, smoking, energy, } \\
\text { diabetes, serum level of thyroid- } \\
\text { stimulating hormone, vitamin D, E }\end{array}$ \\
\hline White $(2006)^{(44)}$ & Cohort & USA & $66-82$ & $\begin{array}{l}F \\
M\end{array}$ & $\begin{array}{l}8877 \\
5101\end{array}$ & $\begin{array}{l}\text { F: } 949 \\
\text { M: } 278 \\
\text { F: } 389 \\
\text { M: } 56 \\
\text { F: } 562 \\
\text { M: } 167\end{array}$ & 20 & $\begin{array}{l}\text { Vitamin C } \\
\text { supplement }\end{array}$ & Questionnaire & $\begin{array}{l}\text { Hip fracture } \\
\text { Wrist fracture } \\
\text { Spine fracture }\end{array}$ & Hospital record & Per $\mathrm{mg} / \mathrm{d}$ & $\begin{array}{l}\text { F: } 1 \\
\text { M: } 1 \\
\text { F: } 1 \\
\text { M: } \\
1 \cdot 01 \\
\text { F: } 1 \\
\text { M: } 1\end{array}$ & $\begin{array}{r}1 \cdot 00,1 \cdot 00 \\
0.99,1.01 \\
1.00,1.00 \\
1,1.03 \\
1.00,1.00 \\
0.99,1.01\end{array}$ & & & 6 & Energy \\
\hline Zhang $(2006)^{(49)}$ & $\begin{array}{l}\text { Case- } \\
\text { control }\end{array}$ & USA & $\geq 50$ & $\mathrm{~F} / \mathrm{M}$ & 2564 & 1215 & - & $\begin{array}{l}\text { Dietary vitamin C } \\
\text { intake }\end{array}$ & $\mathrm{FFQ}$ & Hip fracture & Hospital record & Q5 v. Q1 & 0.71 & $0.51,1$ & & & 8 & $\begin{array}{l}\text { Age, sex, BMI, physical activity, } \\
\text { energy, Ca, vitamin D, protein, } \\
\text { caffeine, alcohol intake }\end{array}$ \\
\hline $\begin{array}{l}\text { Martinez-Ramirez } \\
(2007)^{(46)}\end{array}$ & $\begin{array}{l}\text { Case- } \\
\text { control }\end{array}$ & Spain & $\geq 65$ & $\mathrm{~F} / \mathrm{M}$ & 334 & 167 & - & $\begin{array}{l}\text { Dietary vitamin C } \\
\text { intake }\end{array}$ & FFQ & Fracture & Medical report & Q4 v. Q1 & 0.91 & $0.37,2 \cdot 23$ & & & 5 & $\begin{array}{l}\text { Age, sex, energy, Ca intake, home } \\
\text { access, chronic condition, Kantz } \\
\text { index, serum vitamin C }\end{array}$ \\
\hline Key $(2007)^{(40)}$ & Cohort & UK & $20-89$ & $\begin{array}{l}\mathrm{F} \\
\mathrm{M}\end{array}$ & $\begin{array}{r}26749 \\
7947\end{array}$ & $\begin{array}{r}1555 \\
343\end{array}$ & 5.2 & $\begin{array}{l}\text { Dietary vitamin C } \\
\text { intake }\end{array}$ & FFQ & Fracture & Self-report & Q5 v. Q1 & $\begin{array}{l}1.06 \\
1.10\end{array}$ & $\begin{array}{l}0.83,1 \cdot 36 \\
0.65,1 \cdot 88\end{array}$ & & & 5 & $\begin{array}{l}\text { Age, smoking, energy intake, } \\
\text { alcohol, BMl, exercise, material } \\
\text { status, HRT }\end{array}$ \\
\hline Sahni (2009) $)^{(20)}$ & Cohort & USA & $70-80$ & F/M & 958 & 100 & 15 & $\begin{array}{l}\text { Dietary vitamin C } \\
\text { intake } \\
\text { Supplement } \\
\text { vitamin C intake } \\
\text { Total vitamin C } \\
\text { intake }\end{array}$ & $\mathrm{FFQ}$ & Hip fracture & $\begin{array}{l}\text { Medical report } \\
\text { Hospital record }\end{array}$ & $\begin{array}{l}\text { T3 v. T1 } \\
\text { T3 v. T1 } \\
\text { T3 v. T1 }\end{array}$ & $\begin{array}{l}0.79 \\
0.36 \\
0.52\end{array}$ & $\begin{array}{l}0.52,1.46 \\
0.16,0.79 \\
0.29,0.95\end{array}$ & & & 6 & $\begin{array}{l}\text { Age, sex, energy intake, oestrogen } \\
\text { use, BMI, multi-vitamin use, height }\end{array}$ \\
\hline Samieri $(2013)^{(41)}$ & Cohort & France & $68-95$ & $F / M$ & 1482 & 155 & 8 & $\begin{array}{l}\text { Dietary vitamin C } \\
\text { intake }\end{array}$ & FFQ & Fracture & Self-report & $\begin{array}{l}\text { Fracture } \\
\text { No-fracture }\end{array}$ & & & $\begin{array}{l}81 \cdot 4 \\
83.2\end{array}$ & $\begin{array}{l}55.5 \\
61.4\end{array}$ & 5 & - \\
\hline Sun $(2014)^{(19)}$ & $\begin{array}{l}\text { Case- } \\
\text { control }\end{array}$ & China & $62-79$ & F/M & 1452 & 726 & - & $\begin{array}{l}\text { Dietary vitamin C } \\
\text { intake }\end{array}$ & FFQ & Hip fracture & $\begin{array}{l}\text { Radiology } \\
\text { photography }\end{array}$ & Q4 v. Q1 & 0.39 & $0.28,0.56$ & & & 5 & $\begin{array}{l}\text { Age, sex, drugs, BMI, education, } \\
\text { occupation, income, family history } \\
\text { of fracture, smoking, alcohol, Ca } \\
\text { and multi-vitamin supplement, } \\
\text { physical activity, energy intake }\end{array}$ \\
\hline Finck $(2015)^{(22)}$ & Cohort & UK & $39-79$ & $\begin{array}{l}\mathrm{F} \\
\mathrm{M}\end{array}$ & $\begin{array}{l}2525 \\
1842 \\
2334 \\
1808\end{array}$ & $\begin{array}{r}339 \\
112 \\
124 \\
78\end{array}$ & 12.6 & $\begin{array}{l}\text { Dietary vitamin C } \\
\text { intake }\end{array}$ & $\begin{array}{l}\text { 7-d Food } \\
\text { record }\end{array}$ & $\begin{array}{l}\text { Hip fracture } \\
\text { Spine fracture }\end{array}$ & & $\begin{array}{l}\text { Q5 v. Q1 } \\
\text { Q5 v. Q1 }\end{array}$ & $\begin{array}{l}1.33 \\
0.64 \\
0.90 \\
1.05\end{array}$ & $\begin{array}{l}0.88,2.00 \\
0.34,1.23 \\
0.50,1.61 \\
0.52,2.13\end{array}$ & & & 7 & $\begin{array}{l}\text { Age, family history of osteoporosis, } \\
\text { BMI, smoking, physical activity, } \\
\text { steroid medication, energy, Ca } \\
\text { intake, Ca and vitamin D } \\
\text { supplemental status, HRT }\end{array}$ \\
\hline
\end{tabular}

F, female; M, male; Q, quantiles; T, tertiles; HRT, hormone replacement therapy. 
Table 3. Characteristics of studies that reported the relationship between vitamin $\mathrm{C}$ intake and risk of osteoporosis (OR, relative risk (RR) and $95 \%$ confidence intervals; $\beta$-coefficients with their standard errors)

\begin{tabular}{|c|c|c|c|c|c|c|c|c|c|c|c|c|c|c|c|c|c|c|}
\hline Author (year) & $\begin{array}{l}\text { Study } \\
\text { design }\end{array}$ & Country & $\begin{array}{c}\text { Age } \\
\text { range } \\
\text { (years) }\end{array}$ & Sex & $n$ & $\begin{array}{l}\text { Number } \\
\text { of cases }\end{array}$ & $\begin{array}{l}\text { Duration of } \\
\text { follow-up } \\
\text { (years) }\end{array}$ & $\begin{array}{l}\text { Exposure } \\
\text { variable }\end{array}$ & $\begin{array}{l}\text { Exposure } \\
\text { assessment }\end{array}$ & $\begin{array}{l}\text { Outcome } \\
\text { variable }\end{array}$ & $\begin{array}{l}\text { Outcome } \\
\text { assessment }\end{array}$ & Comparison & $\begin{array}{l}\text { OR } \\
\text { or } \\
\text { RR }\end{array}$ & $95 \% \mathrm{Cl}$ & $\beta$ & SE & $\begin{array}{l}\text { Study } \\
\text { quality }\end{array}$ & Matching or adjustments \\
\hline $\begin{array}{l}\text { Park } \\
(2011)^{(26)}\end{array}$ & $\begin{array}{l}\text { Case- } \\
\text { control }\end{array}$ & $\begin{array}{l}\text { South } \\
\text { Korea }\end{array}$ & $50-70$ & $\mathrm{~F}$ & 144 & 72 & - & $\begin{array}{l}\text { Dietary } \\
\text { vitamin C }\end{array}$ & $\mathrm{FFQ}$ & $\begin{array}{l}\text { Osteoporosis } \\
(<-2.5 \text { T-score/ } \\
\text { LS-FN-FT) }\end{array}$ & DXA (Lunar) & Q4 v. Q1 & 0.653 & $0.187,2 \cdot 279$ & & & 5 & $\begin{array}{l}\text { Energy intake, age, BMI, } \\
\text { HRT/ age-matching }\end{array}$ \\
\hline $\begin{array}{l}\text { Zhang } \\
\text { (2015) }^{(49)}\end{array}$ & $\begin{array}{l}\text { Cross- } \\
\text { sectional }\end{array}$ & China & $30-90$ & $\mathrm{~F}$ & 1878 & 529 & - & $\begin{array}{l}\text { Dietary } \\
\text { vitamin C }\end{array}$ & FFQ & $\begin{array}{l}\text { Osteoporosis } \\
\text { (<-2.5 T-score/ } \\
\text { Cal) }\end{array}$ & QUS (Hologic) & Per 1 unit & 1.369 & $1.037,1.817$ & 0.313 & 0.146 & 6 & - \\
\hline $\begin{array}{l}\mathrm{Kim} M H \\
(2016)^{(21)}\end{array}$ & $\begin{array}{l}\text { Cross- } \\
\text { sectional }\end{array}$ & $\begin{array}{l}\text { South } \\
\text { Korea }\end{array}$ & $\geq 50$ & $\mathrm{~F} / \mathrm{M}$ & 3047 & 1212 & - & $\begin{array}{l}\text { Dietary } \\
\text { vitamin C }\end{array}$ & 24-h recall & $\begin{array}{l}\text { Osteoporosis } \\
(<-2.5 \text { T-score/ } \\
\text { LS-FN-TH) }\end{array}$ & DXA (Hologic) & Q4 v. Q1 & 0.67 & $0.47,0.97$ & & & 8 & $\begin{array}{l}\text { Age, sex, income, } \\
\text { education, smoking, HRT, } \\
\text { survey year, energy intake, } \\
\text { Ca intake, blood vitamin D } \\
\text { level }\end{array}$ \\
\hline $\begin{array}{l}\text { Sugiura } \\
(2016)^{(43)}\end{array}$ & Cohort & Japan & $30-70$ & $\mathrm{~F}$ & 187 & 17 & 4 & $\begin{array}{l}\text { Dietary } \\
\text { vitamin C }\end{array}$ & FFQ & $\begin{array}{l}\text { Osteoporosis } \\
\text { (T-sore exceeded } \\
70 \% / F A)\end{array}$ & DXA (ALOKA) & T3 v. T1 & 0.7 & $0.01,1.17$ & & & 6 & $\begin{array}{l}\text { Age, weight, height, years } \\
\text { since menopause, current } \\
\text { tobacco use, alcohol } \\
\text { intake, exercise habit, } \\
\text { supplement use, energy } \\
\text { intake, intake of } \mathrm{Ca}, \mathrm{Mg}, \mathrm{K} \text {, } \\
\text { vitamin D }\end{array}$ \\
\hline
\end{tabular}

F, female; M, male; LS, lumber spine; FN, femur neck; FT, femoral total; Q, quantiles; HRT, hormone replacement therapy; TH, total hip; CAL, calcaneus; FA, forearm. 
coefficient $^{(24,25,27-29,31,38,42,50,51,54,58,59,61)}$; those that reported BMD's means and standard deviations in categories of vitamin $\mathrm{C}$ intake $^{(55)}$ or users vitamin C supplement $v$. non-users ${ }^{(52)}$; those that reported relative risks (RR) and $95 \% \mathrm{CI}$ in tertiles of vitamin C intake ${ }^{(57)}$; and those that measured BMD at calcaneus ${ }^{(56)}$, total forearm, trabecular forearm, cortical forearm ${ }^{(53)}$ and whole body ${ }^{(30)}$. Finally, four studies ${ }^{(23,26,34,60)}$ that examined the correlation between dietary vitamin $\mathrm{C}$ intake and BMD at femoral neck (four effect sizes obtained from four studies) and lumbar spine (three effect sizes obtained from three studies) were included in the meta-analysis. Studies that reported correlation coefficient between dietary vitamin $\mathrm{C}$ intake and BMD included 3529 individuals in total. The meta-analysis of four effect sizes obtained from four studies at the femoral neck indicated that greater dietary vitamin $\mathrm{C}$ intake was significantly associated with BMD (Fisher's Z: 0.18; 95\% CI 0.06, 0.30, P 0.003). Although between-study heterogeneity was statistically significant $\left(I^{2}=87.6 \%\right.$; $\left.P_{\text {heterogeneity }}<0.001\right)$, we did not perform subgroup analysis to find the source of heterogeneity owing to the low number of publications. Combining three effect sizes obtained from three studies at lumbar spine indicated that greater dietary vitamin $\mathrm{C}$ intake was significantly associated with BMD (Fisher's $Z=0.14 ; 95 \%$ CI 0.06, 0.22, $P$ 0.001). Fisher's $Z$ and $95 \%$ CI that was calculated in meta-analysis is equal to the correlation coefficient and $95 \%$ CI. No evidence of between-study heterogeneity was found $\left(I^{2}=30.7 \%\right.$; $\left.P_{\text {heterogeneity }}=0.236\right)$ (Fig. 2$)$. Begg's test $(P=0.53)$ and Egger's test $(P=0 \cdot 07)$ showed no publication bias.

Vitamin c intake and risk of fracture. Of thirteen studies that were in the systematic review, we excluded seven studies from the meta-analysis: those that considered total fracture as the outcome ${ }^{(40,42,46)}$, supplementary vitamin $\mathrm{C}$ as an exposure ${ }^{(44)}$, mean and standard deviation of vitamin $\mathrm{C}$ intake in fracture and control groups $^{(41,45)}$ and those that reported the risk of hip fracture for vitamin C consumption per $4184 \mathrm{~kJ} / \mathrm{d}(1000 \mathrm{kcal} / \mathrm{d})^{(39)}$. Finally, six studies that considered hip fracture were included in the metaanalysis ${ }^{(19,20,22,47-49)}$. Publications that examined the association between vitamin $\mathrm{C}$ intake and risk of fracture included 10810 individuals with 2898 cases of hip fractures. In this meta-analysis, nine effect sizes, obtained from six studies, indicated that high levels of vitamin $\mathrm{C}$ intake were not significantly associated with risk of hip fractures (overall $\mathrm{RR}=0.74 ; 95 \% \mathrm{CI} 0 \cdot 51,1 \cdot 08, P$ 0.001) (Fig. 3). Significant between-study heterogeneity was found $\left(I^{2}=79.1 \% ; \quad P_{\text {heterogeneity }}<0.001\right)$. Subgroup analysis was performed to find the potential sources of heterogeneity. This analysis indicated that study design, sex and age were the main sources of between-study heterogeneity (Table 4). In cohort and case-control studies, non-significant associations were found. However, between-study heterogeneity was not apparent in cohort studies. In males, dietary vitamin $\mathrm{C}$ intake was significantly associated with hip fracture (overall $\mathrm{RR}=0.47 ; 95 \%$ CI $0.28,0.79, P$ 0.004). In females, a non-significant association was seen (overall $\mathrm{RR}=0 \cdot 90$; $95 \%$ CI $0.71,1 \cdot 15, P 0.394$ ). In both females and males, $29 \%$ decreased incidence of hip fracture was seen by greater dietary vitamin $\mathrm{C}$ intake (overall $\mathrm{RR}=0.71 ; 95 \% \mathrm{CI}$ 0.56, 0.92, P 0.009). The association between dietary vitamin $\mathrm{C}$ intake and hip fracture was significant in participants aged 70 years or older (overall $\mathrm{RR}=0.72 ; 95 \%$ CI $0.57,0.92, P$ 0.009). No publication bias was found (Begg's test $P=0.74$ and Egger's test $P=0.83$ ).

Vitamin C intake and risk of osteoporosis. Out of four included studies in the systematic review, one study was excluded from the meta-analysis ${ }^{(61)}$. This study reported the risk of osteoporosis for one unit vitamin C consumption. Three studies considered the risk of osteoporosis in categorised of vitamin $\mathrm{C}$ intake which were included in the metaanalysis $^{(21,26,43)}$. These studies included 3378 individuals with

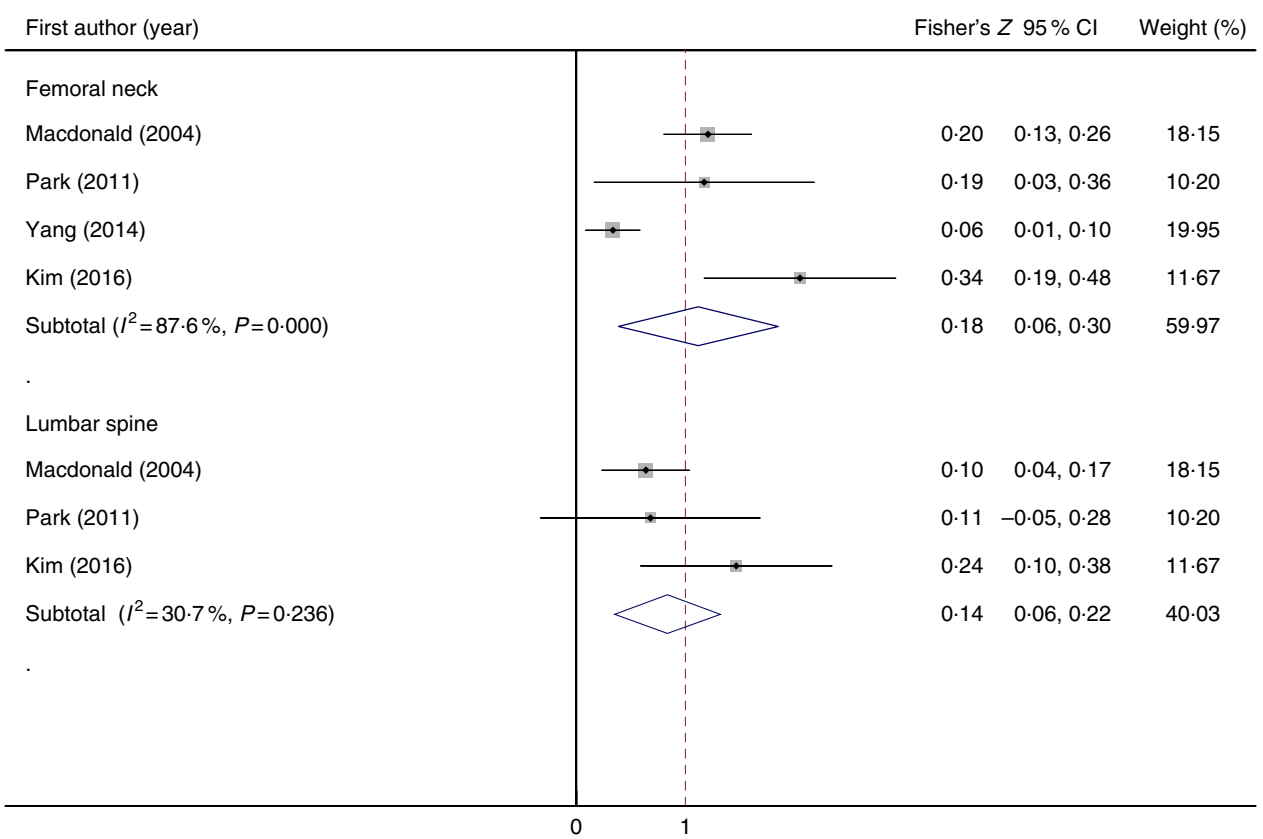

Fig. 2. Forest plot of correlation coefficient in bone mineral density at the femoral neck and lumbar spine and dietary vitamin $\mathrm{C}$ intake. 


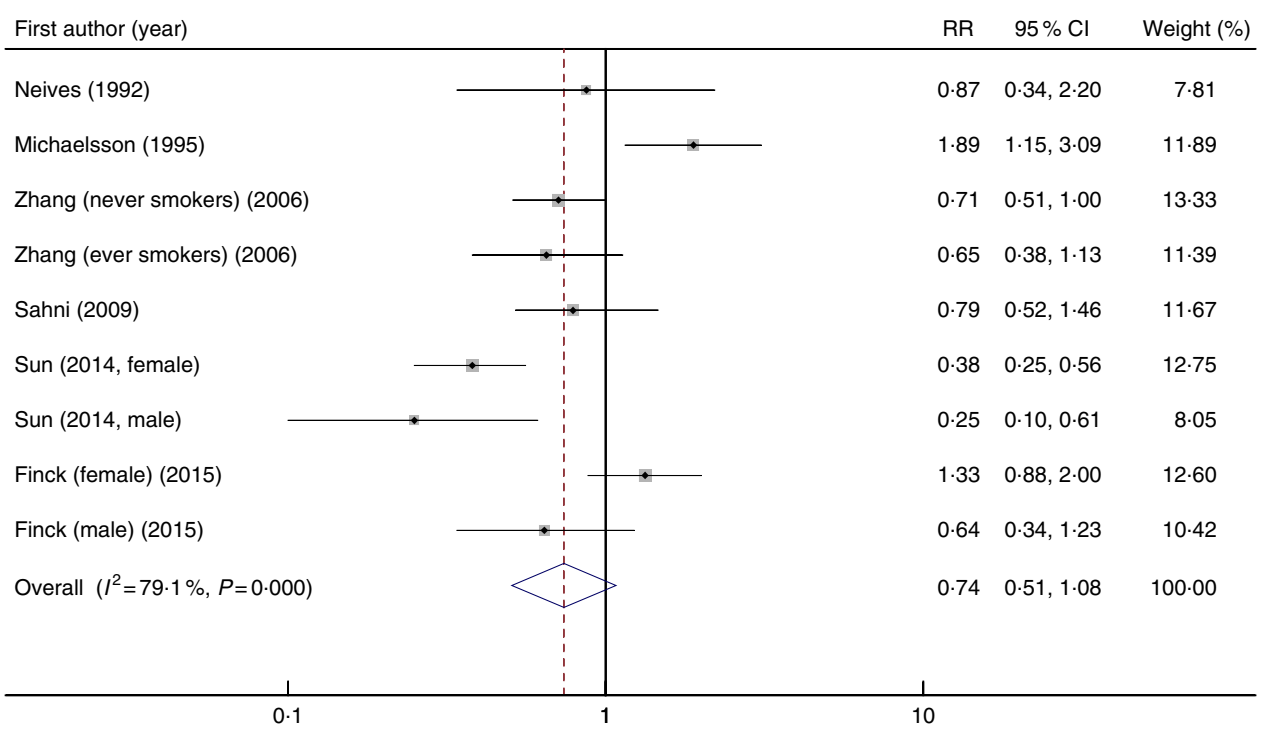

Fig. 3. Forest plot of the association between dietary vitamin $C$ intake and the risk of hip fracture. RR, relative risk.

Table 4. Subgroup analysis of dietary vitamin $C$ intake and risk of hip fracture (Relative risks (RR) and $95 \%$ confidence intervals)

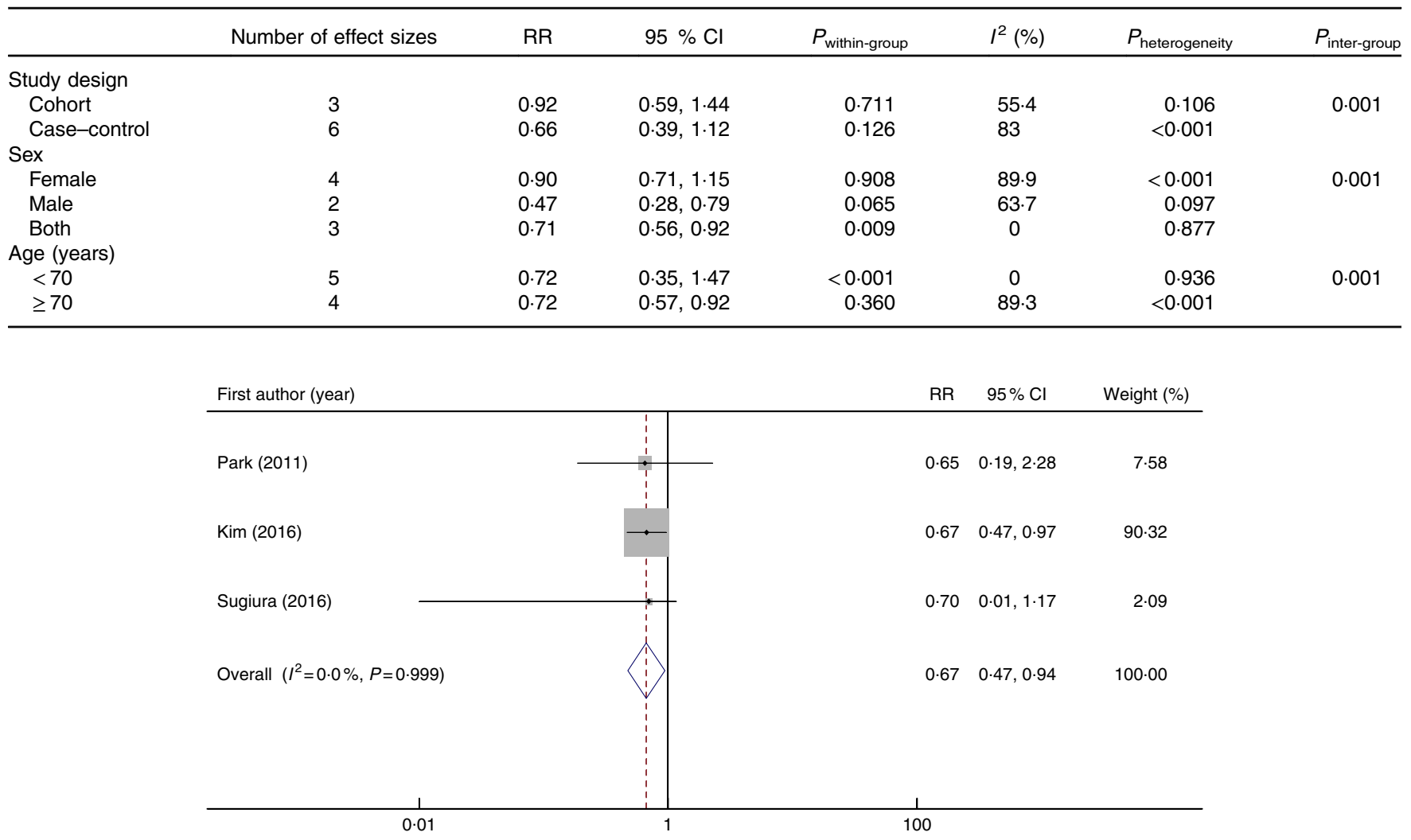

Fig. 4. Forest plot of the association between dietary vitamin $C$ intake and the risk of osteoporosis. RR, relative risk.

1301 cases of osteoporosis. In the meta-analysis of effect sizes obtained from three included studies, we found that higher vitamin $\mathrm{C}$ intake was inversely associated with the risk of osteoporosis (overall RR $=0.67 ; 95 \%$ CI 0.47, 0.94, P: 0.022; $\left.I^{2}=0 \% ; P_{\text {heterogeneity }}=0.999\right)$ (Fig. 4). No publication bias was found (Begg's test $P=0.60$ and Egger's test $P=0.95$ ).

\section{Discussion}

In this meta-analysis, we found that greater dietary intake of vitamin $\mathrm{C}$ was positively associated with a higher BMD at the femoral neck and lumbar spine. In addition, greater dietary vitamin $\mathrm{C}$ intake was significantly associated with reduced risk 
of hip fracture and osteoporosis. This is the first meta-analysis that examined the association of dietary vitamin $\mathrm{C}$ intake with risk of fracture, osteoporosis and BMD.

Osteoporosis is a chronic condition that affects a large number of elderly people ${ }^{(62)}$. The incidence of fracture has increased recently in parallel to ageing throughout the world $^{(63)}$. Among several factors that might influence the risk of fractures and osteoporosis, dietary intakes are of great importance. Vitamin C is essential for collagen synthesis and osteoblastogenesis. Ascorbic acid specifically stimulated type I and III collagen synthesis ${ }^{(64-67)}$. In addition, vitamin C deficiency stimulates osteoclastogenesis ${ }^{(68)}$. During osteoclastogenesis, ascorbic acid acts as an oxidant, first stimulating osteoclast formation, later limiting osteoclast life span. This action results in adaptation of osteoclastogenesis ${ }^{(68,69)}$. Although vitamin $\mathrm{C}$ indeed has a major effect on collagen synthesis, the main and most crucial effect is on osteoblast differentiation. Vitamin C can prevent the loss of osteoblast differentiation markers (Osterix, osteocalcin, runt-related transcription 2, bone morphogenetic protein 2) and attenuate bone loss, as well as stimulate bone formation $^{(70)}$. Vitamin $\mathrm{C}$ actually is a marker of dietary fruit and vegetable intake, healthy dietary patterns and total antioxidant intake. Greater intake of fruit, vegetables and antioxidants, and healthier dietary pattern, was associated with bone health ${ }^{(71,72)}$.

We found that greater intakes of dietary vitamin $\mathrm{C}$ were associated with higher BMD at femoral neck and lumbar spine. Previous studies have reached the same findings ${ }^{(23-27,29,34,52,60)}$. However, some investigations indicated no association between dietary vitamin $\mathrm{C}$ intake and BMD's correlation coefficient at the femoral neck and lumbar spine ${ }^{(38,58,59)}$. Such findings might be explained by the validity of the FFQ used in different studies and lack of controlling for several confounders. However, it must be noted that these correlations are considered as weak correlations.

In terms of hip fracture, we found a significant inverse association between greater dietary vitamin $\mathrm{C}$ intake and the risk of hip fracture in both males and females. Although these findings were in agreement with previous publications ${ }^{(19,20)}$, other studies have found a non-significant association between dietary vitamin $\mathrm{C}$ intake and the risk of hip fracture ${ }^{(22,39,40,42,44,46-49)}$. These findings might be explained by different selection bias, different study designs and sample sizes, as well as lack of controlling for potential confounders and the validity of the FFQ used in different studies.

Another finding of our study was that dietary intakes of vitamin $\mathrm{C}$ were inversely associated with the risk of osteoporosis. Our finding was in agreement with that of the study by Kim \& Lee ${ }^{(21)}$. Although other publications in this regard indicated non-significant or direct associations ${ }^{(26,43,61)}$, such findings might be explained by the lack of controlling for potential confounders, low sample sizes and low incidence of osteoporosis during follow-up.

Although the present study is the first meta-analysis that examined the association of dietary vitamin $\mathrm{C}$ intake and BMD, fracture or osteoporosis, it has some limitations that should be considered. Searching was limited to published articles. Although there was no evidence of publication bias, lack of considering unpublished studies might have influenced the findings. Some studies reported the $\beta$-correlation coefficient and we did not include them in the meta-analysis. Owing to the cross-sectional design of most studies that reported mean BMD, our findings in this regard cannot indicate causality. Differences in study design lead to differences in their reliability. We could not consider this point because of the lack of enough studies in this regard. Although significant heterogeneity was seen, due to lack of enough studies, we could not perform subgroup analysis.

In conclusion, we found that greater dietary vitamin $\mathrm{C}$ intake was associated with higher BMD at the femoral neck and lumbar spine. In addition, reduced risk of hip fracture and osteoporosis were associated with greater dietary vitamin $\mathrm{C}$ intakes. However, some questions still need to be answered to determine causality. Further prospective cohort studies with long duration of follow-up, valid instruments for measurement of dietary vitamin C, BMD, fracture and osteoporosis are warranted to support the relation between dietary vitamin $\mathrm{C}$ and $\mathrm{BMD}$, risk of fracture and osteoporosis.

\section{Acknowledgements}

This study was supported by the Tehran University of Medical Sciences, Tehran, Iran.

The authors' contributions were as follows: H. M., S. S.-b. and K. D. designed the research; H. M. conducted the research and performed statistical analysis; H. M. and S. S.-b. wrote the paper; and S. S.-b. had responsibility for final content. All authors read and approved the final manuscript.

The authors declare that there are no conflicts of interest.

\section{References}

1. National Institutes of Health (NIH) Osteoporosis and Related Bone Diseases National Resource Center (2015) Osteoporosis overview. Bethesda, MD: NIH. https://www.bones.nih.gov/ health-info/bone/osteoporosis/overview (accessed March 2018).

2. Kanis JA, Melton LJ 3rd, Christiansen C, et al. (1994) The diagnosis of osteoporosis. J Bone Miner Res 9, 1137-1141.

3. World Health Organization (1994) Assessment of Fracture Risk and its Application to Screening for Postmenopausal Osteoporosis. Report of a WHO Study Group. WHO Technical Report Series, no. 843. Geneva: WHO.

4. Johnell O \& Kanis JA (2006) An estimate of the worldwide prevalence and disability associated with osteoporotic fractures. Osteoporos Int 17, 1726-1733.

5. Becker DJ, Kilgore ML \& Morrisey MA (2010) The societal burden of osteoporosis. Curr Rheumatol Rep 12, 186-191.

6. Liem IS, Kammerlander C, Suhm N, et al. (2014) Literature review of outcome parameters used in studies of Geriatric Fracture Centers. Arch Orthop Trauma Surg 134, 181-187.

7. Richmond J, Aharonoff GB, Zuckerman JD, et al. (2003) Mortality risk after hip fracture. J Orthop Trauma 17, 53-56.

8. Anagnostis P, Karagiannis A, Kakafika AI, et al. (2009) Atherosclerosis and osteoporosis: age-dependent degenerative processes or related entities? Osteoporos Int 20, 197-207.

9. Bollet AJ, Engh G \& Parson W (1965) Epidemiology of osteoporosis; sex and race incidence of hip fractures. Arch Intern Med 116, 191-194.

10. Melton LJ 3rd (2003) Epidemiology worldwide. Endocrinol Metab Clin North Am 32, 1-13.

11. Raisz LG (2005) Pathogenesis of osteoporosis: concepts, conflicts, and prospects. J Clin Invest 115, 3318-3325. 
12. Ojo F, Al Snih S, Ray LA, et al. (2007) History of fractures as predictor of subsequent hip and nonhip fractures among older Mexican Americans. J Natl Med Assoc 99, 412-418.

13. Simonelli C, Cohan D, Kopher R, et al. (2006) Health Care Guideline: Diagnosis and Treatment of Osteoporosis, 5th ed. Bloomington, MN: Institute for Clinical Systems Improvement.

14. Wong PK, Christie JJ \& Wark JD (2007) The effects of smoking on bone health. Clin Sci (Lond) 113, 233-241.

15. Berg KM, Kunins HV, Jackson JL, et al. (2008) Association between alcohol consumption and both osteoporotic fracture and bone density. Am J Med 121, 406-418.

16. Ilich JZ \& Kerstetter JE (2000) Nutrition in bone health revisited: a story beyond calcium. J Am Coll Nutr 19, 715-737.

17. Feskanich D, Willett WC, Stampfer MJ, et al. (1996) Protein consumption and bone fractures in women. Am J Epidemiol 143, 472-479.

18. Tucker KL, Morita K, Qiao N, et al. (2006) Colas, but not other carbonated beverages, are associated with low bone mineral density in older women: The Framingham Osteoporosis Study. Am J Clin Nutr 84, 936-942.

19. Sun LL, Li BL, Xie HL, et al. (2014) Associations between the dietary intake of antioxidant nutrients and the risk of hip fracture in elderly Chinese: a case-control study. Br J Nutr 112, 1706-1714.

20. Sahni S, Hannan MT, Gagnon D, et al. (2009) Protective effect of total and supplemental vitamin $\mathrm{C}$ intake on the risk of hip fracture-a 17-year follow-up from the Framingham Osteoporosis Study. Osteoporos Int 20, 1853-1861.

21. Kim MH \& Lee HJ (2016) Osteoporosis, vitamin C intake, and physical activity in Korean adults aged 50 years and over. J Phys Ther Sci 28, 725-730.

22. Finck H, Hart AR, Lentjes MAH, et al. (2015) Cross-sectional and prospective associations between dietary and plasma Vitamin C, heel bone ultrasound, and fracture risk in men and women in the European Prospective Investigation into Cancer in Norfolk cohort. Am J Clin Nutr 102, 1416-1424.

23. Kim DE, Cho SH, Park HM, et al. (2016) Relationship between bone mineral density and dietary intake of -carotene, vitamin $\mathrm{C}$, zinc and vegetables in postmenopausal Korean women: a cross-sectional study. I Int Med Res 44, 1103-1114.

24. Liu ZM, Leung J, Wong SYS, et al. (2015) Greater fruit intake was associated with better bone mineral status among Chinese elderly men and women: results of Hong Kong Mr. Os and Ms. Os studies. J Am Med Dir Assoc 16, 309-315.

25. Kim YA, Kim KM, Lim S, et al. (2015) Favorable effect of dietary vitamin $\mathrm{C}$ on bone mineral density in postmenopausal women (KNHANES IV, 2009): discrepancies regarding skeletal sites, age, and vitamin D status. Osteoporos Int 26, 2329-2337.

26. Park HM, Heo J \& Park Y (2011) Calcium from plant sources is beneficial to lowering the risk of osteoporosis in postmenopausal Korean women. Nutr Res 31, 27-32.

27. Prynne CJ, Mishra GD, O'Connell MA, et al. (2006) Fruit and vegetable intakes and bone mineral status: a cross sectional study in 5 age and sex cohorts. Am J Clin Nutr 83, 1420-1428.

28. Ilich JZ, Brownbill RA \& Tamborini L (2003) Bone and nutrition in elderly women: protein, energy, and calcium as main determinants of bone mineral density. Eur J Clin Nutr 57, 554-565.

29. Hall SL \& Greendale GA (1998) The relation of dietary vitamin C intake to bone mineral density: Results from the PEPI study. Calcif Tissue Int 63, 183-189.

30. Casale M, von Hurst PR, Beck KL, et al. (2016) Lean mass and body fat percentage are contradictory predictors of bone mineral density in pre-menopausal pacific island women. Nutrients 8, 470-481.
31. Leveille SG, LaCroix AZ, Koepsell TD, et al. (1997) Dietary vitamin $\mathrm{C}$ and bone mineral density in postmenopausal women in Washington State, USA. J Epidemiol Community Health 51, 479-485.

32. Moher D, Liberati A, Tetzlaff J, et al. (2009) Preferred reporting items for systematic reviews and meta-analyses: the PRISMA statement. PLOS Med 6, e1000097.

33. New SA, Bolton-Smith C, Grubb DA, et al. (1997) Nutritional influences on bone mineral density: a cross-sectional study in premenopausal women. Am J Clin Nutr 65, 1831-1839.

34. Macdonald HM, New SA, Golden MHN, et al. (2004) Nutritional associations with bone loss during the menopausal transition: evidence of a beneficial effect of calcium, alcohol, and fruit and vegetable nutrients and of a detrimental effect of fatty acids. Am J Clin Nutr 79, 155-165.

35. Wells GA, Shea B, O'Connell D, et al. (2014) The NewcastleOttawa Scale (NOS) for assessing the quality of nonrandomised studies in meta-analyses. Ottawa, Canada: Ottawa Hospital Research Institute. http://www.ohri.ca/programs/ clinical_epidemiology/oxford.asp (accessed June 2016).

36. Egger M, Smith GD \& Altman DG (2008) Systematic Reviews in Health Care: Meta-Analysis in Context, 2th ed. London: BMJ Publishing Group.

37. Higgins JP, Thompson SG, Deeks JJ, et al. (2003) Measuring inconsistency in meta-analyses. BMJ 327, 557-560.

38. Chan R, Woo J \& Leung J (2011) Effects of food groups and dietary nutrients on bone loss in elderly Chinese population. J Nutr Health Aging 15, 287-294.

39. Holbrook TL, Barrett-Connor E \& Wingard DL (1988) Dietary calcium and risk of hip fracture: 14-year prospective population study. Lancet ii, 1046-1049.

40. Key TJ, Appleby PN, Spencer EA, et al. (2007) Calcium, diet and fracture risk: a prospective study of 1898 incident fractures among 34696 British women and men. Public Health Nutr 10, 1314-1320.

41. Samieri C, Ginder Coupez V, Lorrain S, et al. (2013) Nutrient patterns and risk of fracture in older subjects: results from the Three-City Study. Osteoporos Int 24, 1295-1305.

42. Simon JA \& Hudes ES (2001) Relation of ascorbic acid to bone mineral density and self-reported fractures among US adults. Am J Epidemiol 154, 427-433.

43. Sugiura M, Nakamura M, Ogawa K, et al. (2016) High vitamin C intake with high serum beta-cryptoxanthin associated with lower risk for osteoporosis in post-menopausal Japanese female subjects: Mikkabi cohort study. J Nutr Sci Vitaminol 62, 185-191.

44. White SC, Atchison KA, Gornbein JA, et al. (2006) Risk factors for fractures in older men and women: The Leisure World Cohort Study. Gend Med 3, 110-123.

45. Lumbers M, New SA, Gibson S, et al. (2001) Nutritional status in elderly female hip fracture patients: comparison with an age-matched home living group attending day centres. $\mathrm{Br} J$ Nutr 85, 733-740.

46. Martínez-Ramírez MJ, Pérez SP, Delgado-Martínez AD, et al. (2007) Vitamin $C$, vitamin $B_{12}$, folate and the risk of osteoporotic fractures. A case-control study. Int J Vitam Nutr Res 77, 359-368.

47. Michaelsson K, Holmberg L, Mallmin H, et al. (1995) Diet and hip fracture risk: a case-control study. Study Group of the Multiple Risk Survey on Swedish Women for Eating Assessment. Int J Epidemiol 24, 771-782.

48. Nieves JW, Grisso JA \& Kelsey JL (1992) A case-control study of hip fracture: evaluation of selected dietary variables and teenage physical activity. Osteoporos Int 2, 122-127.

49. Zhang J, Munger RG, West NA, et al. (2006) Antioxidant intake and risk of osteoporotic hip fracture in Utah: an effect modified by smoking status. Am J Epidemiol 163, 9-17. 
50. Hernández-Avila M, Stampfer MJ, Ravnikar VA, et al. (1992) Caffeine and other predictors of bone density among pre- and perimenopausal women. Epidemiology 4, 128-134.

51. Kaptoge S, Welch A, McTaggart A, et al. (2003) Effects of dietary nutrients and food groups on bone loss from the proximal femur in men and women in the 7th and 8th decades of age. Osteoporos Int 14, 418-428.

52. Morton DJ, Barrett-Connor EL \& Schneider DL (2001) Vitamin C supplement use and bone mineral density in postmenopausal women. J Bone Miner Res 16, 135-140.

53. New SA, Robins SP, Campbell MK, et al. (2000) Dietary influences on bone mass and bone metabolism: Further evidence of a positive link between fruit and vegetable consumption and bone health? Am J Clin Nutr 71, 142-151.

54. Rivas A, Romero A, Mariscal-Arcas M, et al. (2012) Association between dietary antioxidant quality score (DAQs) and bone mineral density in Spanish women. Nutr Hosp 27, 1886-1893.

55. Sahni S, Hannan MT, Gagnon D, et al. (2008) High vitamin C intake is associated with lower 4-year bone loss in elderly men. J Nutr 138, 1931-1938.

56. Sasaki S \& Yanagibori R (2001) Association between current nutrient intakes and bone mineral density at calcaneus in preand postmenopausal Japanese women. J Nutr Sci Vitaminol 47, 289-294.

57. Sugiura M, Nakamura M, Ogawa K, et al. (2011) Dietary patterns of antioxidant vitamin and carotenoid intake associated with bone mineral density: Findings from post-menopausal Japanese female subjects. Osteoporos Int 22, 143-152.

58. Wang MC, Villa ML, Marcus R, et al. (1997) Associations of vitamin $\mathrm{C}$, calcium and protein with bone mass in postmenopausal Mexican American women. Osteoporos Int 7, 533-538.

59. Wolf RL, Cauley JA, Pettinger M, et al. (2005) Lack of a relation between vitamin and mineral antioxidants and bone mineral density: results from the Women's Health Initiative. Am J Clin Nutr 82, 581-588.

60. Yang YJ \& Kim J (2014) Factors in relation to bone mineral density in korean middle-aged and older men: 2008-2010
Korea National Health and Nutrition Examination Survey. Ann Nutr Metab 64, 50-59.

61. Zhang J, Zhang K, Shi H, et al. (2015) A cross-sectional study to evaluate the associations between hypertension and osteoporosis in Chinese postmenopausal women. Int J Clin Exp Med 8, 21194-21200.

62. Liu K, Liu P, Liu R, et al. (2015) Relationship between serum leptin levels and bone mineral density: a systematic review and meta-analysis. Clin Chim Acta 444, 260-263.

63. Melton LJ, Kearns AE, Atkinson EJ, et al. (2009) Secular trends in hip fracture incidence and recurrence. Osteoporos Int 20, 687-694.

64. Carinci F, Pezzetti F, Spina AM, et al. (2005) Effect of Vitamin C on pre-osteoblast gene expression. Arch Oral Biol 50, 481-496.

65. Harada S, Matsumoto T \& Ogata E (1991) Role of ascorbic acid in the regulation of proliferation in osteoblast-like MC3T3E1 cells. J Bone Miner Res 6, 903-908.

66. Chan D, Lamande SR, Cole WG, et al. (1990) Regulation of procollagen synthesis and processing during ascorbateinduced extracellular matrix accumulation in vitro. Biochem J 269, 175-181.

67. Barnes MJ (1975) Function of ascorbic acid in collagen metabolism. Ann N Y Acad Sci 258, 264-277.

68. Hie M \& Tsukamoto I (2011) Vitamin C-deficiency stimulates osteoclastogenesis with an increase in RANK expression. J Nutr Biochem 22, 164-171.

69. Le Nihouannen D, Barralet JE, Fong JE, et al. (2010) Ascorbic acid accelerates osteoclast formation and death. Bone $\mathbf{4 6}$, 1336-1343.

70. Aghajanian P, Hall S, Wongworawat MD, et al. (2015) The roles and mechanisms of actions of vitamin $\mathrm{C}$ in bone: new developments. J Bone Miner Res 30, 1945-1955.

71. Ward KA, Prentic A, Kuh DL, et al. (2016) Life course dietary patterns and bone health in later life in a British Birth Cohort Study. J Bone Miner Res 31, 1167-1176.

72. Sahni S, Mangano KM, McLean RR, et al. (2015) Dietary approaches for bone health: lessons from the Framingham Osteoporosis Study. Curr Osteoporos Rep 13, 245-255. 